\title{
Statistical and fractal analyses of autofluorescence of myocardium tissue images in diagnostics of acute coronary insufficiency
}

\author{
O.V. Dubolazov, M.I. Sidor, A.O. Karachevtsev \\ Chernivtsi National University, Optics and Publishing Department, \\ 2, Kotsyubinsky str., 58012 Chernivtsi, Ukraine \\ E-mail:a.dubolazov@chnu.edu.ua
}

\begin{abstract}
This research presents the results of investigation of laser polarization fluorescence of biological layers (histological sections of the myocardium). The polarized structure of autofluorescence imaging layers of biological tissues was detected and investigated. Proposed in this work is the model for describing formation of polarization inhomogeneity in autofluorescent images of biological optically anisotropic layers. On its basis, analytically and experimentally tested is the method of laser autofluorescent polarimetry. Analyzed has been the effectiveness of this method in the postmortem diagnosis of infarction. The objective criteria (statistical moments) of differentiation of autofluorescent images of histological sections myocardium have been determined. The operational characteristics (sensitivity, specificity, accuracy) of this technique have been ascertained.
\end{abstract}

Keywords: autofluorescence, polarization, birefringence, dichroism, statistical optics, diagnostics, autofluorescence imaging, histological sections of the myocardium.

Manuscript received 17.11.14; revised version received 27.02.15; accepted for publication 27.05.15; published online 08.06.15.

\section{Introduction}

Biological tissues represent structurally heterogeneous optical anisotropic media with absorption. To describe interactions of polarized light with these complex systems more generalized approximations are required based on Mueller-matrix formalism. Nowadays, many practical techniques based on the measurement and analysis of Mueller matrices of the investigated samples are applied to biological and medical researches [1-5]. A separate direction - laser polarimetry - was formed in matrix optics in recent 10-15 years [6-10]. On its base, interrelations between the set of statistical moments of the $1^{\text {st }}$ to $4^{\text {th }}$ orders were determined, which characterize the distributions of
Mueller-matrix elements, and the parameters of linear birefringence of fibrillar protein networks in human biological tissues. Using them, diagnostics of pathological changes in skin derma, epithelial and connective tissues of women's reproductive sphere organs, etc., has been realized [11-24].

New step in the development of optical diagnostics methods of biological objects is a combination of polarimetry and fluorescence methods [25].

This work is focused on research of interrelation between optical anisotropy of histological sections taken from myocardium and polarization structure of posthumous autofluorescent images in diagnostics of coronary heart disease (CHD) and acute coronary insufficiency (ACI). 


\section{Brief theory}

In this work, we have limited ourselves by considering the spectral-selective case - luminescence of optically active porphyrins of biological layers in the red $\left(\lambda_{f}=\right.$ $0.63 \ldots 0.65 \mu \mathrm{m})$ spectral range [25]. The excitation of autofluorescence was realized by a blue solid-state laser with the wavelength $\lambda=0.405 \mu \mathrm{m}$ that coincides with the porphyrin's absorption peak.

The following model ideas concerning optical anisotropy of optically thin (extinction coefficient $\tau \prec 0.1$ ) and non-polarizing biological layers are used as the basis for description of laser polarization autofluorescence of biological tissues.

2.1. Absorption - Amino acids and polypeptide chains (primary structure of protein) made by them form the fibrillar (secondary structure) protein networks possessing the linear dichroism. This absorption has liquid crystalline networks of endogenous porphyrins associated with protein molecules. Availability of complex spiral-like structures or their combinations (third-order structure) of polypeptide protein structures forms the circular dichroism. This mechanism is inherent to optically active porphyrin molecules.

2.2. Fluorescence - Polarization appearance of porphyrin fluorescence is characterized by Muellermatrix adduced in [25-28]

$$
\begin{aligned}
& \{F\}=\left\|\begin{array}{cccc}
1 & F_{12} & 0 & 0 \\
F_{21} & F_{22} & 0 & 0 \\
0 & 0 & F_{33} & 0 \\
0 & 0 & 0 & F_{44}
\end{array}\right\| \text {, where } \\
& F_{i k}=F_{11}^{-1}\left\{\begin{array}{l}
F_{11}=a-b \sin ^{2} \vartheta \\
F_{12}=F_{21}=-b \sin ^{2} \vartheta \\
F_{22}=b\left(1+\cos ^{2} \vartheta\right), \\
F_{33}=2 b \cos \vartheta \\
F_{44}=2 c \cos \vartheta
\end{array}\right.
\end{aligned}
$$

Here, $\vartheta$ is the scattering angle; $a$ and $b$ are the interrelated constants for the system of linear oscillators in isotropic medium, defined by following relations:

$$
\begin{aligned}
& a=0.5\left(1+\left\langle\cos ^{2} \varepsilon\right\rangle\right), \\
& b=0.25\left(3\left\langle\cos ^{2} \varepsilon\right\rangle-1\right),
\end{aligned}
$$

where $\varepsilon$ is the angle between the emissive dipole and azimuth of polarization inherent to the exciting beam.

The parameter $c$ is undoubtedly related with optical activity. From the classical viewpoint, a "circular oscillator" would be caused by the induced electric and magnetic transition dipole moments being fully parallel or antiparallel and having the same magnitude. Following this description, optically active liquid-crystal molecules are "elliptical oscillators".
2.3. Phase modulation of fluorescence. Amino acids and polypeptide chains made by them (primary structure of protein) demonstrate optical activity.

Fibrillar (secondary structure) protein networks formed by polypeptide chains possess linear birefringence.

Considering the effect of all mechanisms of optically anisotropic absorption and phase anisotropy, the Mueller matrix of laser polarization autofluorescence of the protein network in biological tissue can be written as follows:

$$
\{M\}=\{D\}\left\{,\{F\}\{\Psi\},\{\Phi\}=\left\|\begin{array}{cccc}
1 & M_{12} & M_{13} & M_{14} \\
M_{21} & M_{22} & M_{23} & M_{24} \\
M_{31} & M_{32} & M_{33} & M_{34} \\
M_{41} & M_{42} & M_{43} & M_{44}
\end{array}\right\|\right. \text {.(4) }
$$

The analysis of matrix (4) shows that elements $M_{i k}$ characterize superposition of mechanisms of linear $\Delta \tau$ and circular $\Delta g$ dichroism; fluorescence of linear $F_{12 ; 21 ; 22 ; 33}$ and elliptical $F_{44}$ oscillators with the following phase modulation of this radiation by optically active molecules $\theta$ and birefringent $\delta$ networks of them.

The "information content" of matrix elements is different. The set of elements $M_{i=1 ; k=1 ; 2 ; 3 ; 4}\left(F_{12}\right)$ characterizes the fluorescence of linear oscillators originated due to the anisotropic absorption. The elements $M_{i=2 ; 3 ; k=1 ; 2 ; 3 ; 4}\left(F_{21 ; 22 ; 33}\right)$ define the phasemodulated $(\delta, \theta)$ fluorescence of linear oscillators. Finally, the values of elements $M_{i=4 ; k=1 ; 2 ; 3 ; 4}\left(F_{21 ; 22 ; 33}, F_{44}\right)$ contain complex information concerning fluorescence of linear $\left(F_{21 ; 2 ; 33}\right)$ and elliptical $\left(F_{44}\right)$ oscillators in optically anisotropic medium with linear and circular birefringence.

The process of forming the polarization structure of the autofluorescence image can be described by the vector parametric equation [14]

$S=\{M\} S_{0}$.

Here $S_{0}, S$ are the Stokes vectors of probing (illuminating) and autofluorescence (polarization modulated) radiation.

From (5), we obtain expressions for the autofluorescence polarization parameters $\alpha^{\otimes}, \beta^{\otimes}$ :

$$
\begin{aligned}
& \alpha^{\otimes}=0.5 \operatorname{arctg} \frac{M_{31}+M_{34}}{M_{21}+M_{24}}, \\
& \beta^{\otimes}=0.5 \arcsin \frac{M_{41}+M_{44}}{1+M_{14}} .
\end{aligned}
$$

\section{Analysis and discussion of experimental results}

\subsection{Measuring the distributions of autofluorescence images polarization parameters}

Experimental measurements were performed in the set of Stokes polarimeter [14] with the use of spectrally selective band-pass light filters (Fig. 1). 


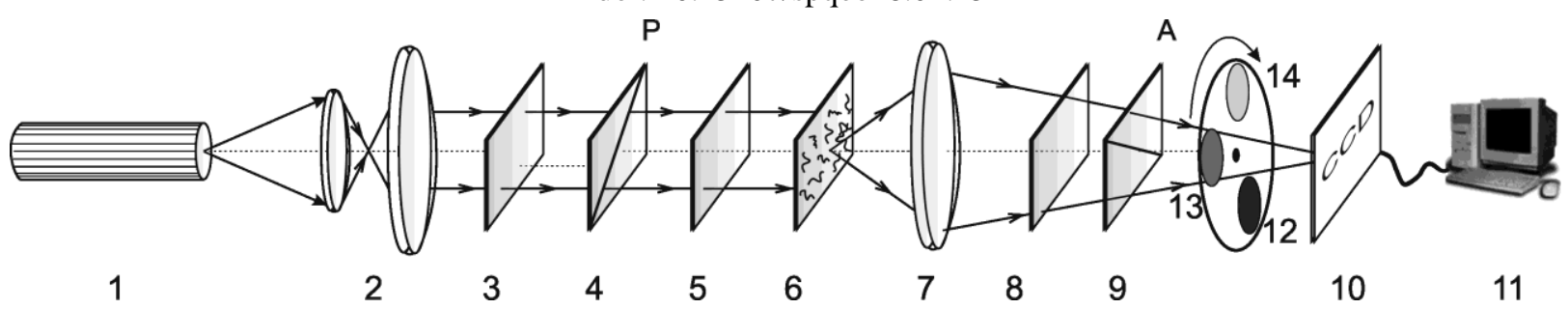

Fig. 1. Optical scheme of Stokes-polarimeter. Explanations are in the text.

To excite autofluorescence, we used the "blue" solid-state laser with the wavelength $\lambda=0.405 \mu \mathrm{m}$ and variable power $W$ (in this research we used $W=50 \mathrm{~mW}$ ). A polarization illuminator consists of quarter-wave plates 3, 5 (Achromatic True Zero-Order Waveplate) and polarizer 4 . The image of the myocardium 6 by means of polarization microscope objective 7 (Nikon CFI Achromat $\mathrm{P}$, focal distance $30 \mathrm{~mm}$, numerical aperture 0.1 , magnification $4 \times$ ) was projected in the plane of lightsensitive CCD-camera 11 (The Imaging Source DMK 41AU02.AS, monochrome 1/2" CCD, Sony ICX205AL (progressive scan), overall amount of pixels $m \times n=$ $1280 \times 960$, light sensitive area size $7600 \times 6200 \mu \mathrm{m}$, sensitivity $0.05 \mathrm{~lx}$, dynamic range 8 bit, deviation of photosensitive characteristics from the linear ones is no more than 12\%). Polarization analysis of coordinate distribution of intensity from the sample 6 was carried out by means of quarter-wave plate 8 and polarizer 9 .

\subsection{Polarization autofluorescence biopsy}

As objects of investigation, two groups of optically thin (extinction coefficient $\tau \approx 0.087 \ldots 0.093$ ) histological sections of biopsy taken from the myocardium inherent to two groups of patients with the following diagnoses:

group 1 - CHD;

group 2 - ACI.

The potentiality of this technique is illustrated by the data presented in Figs. 2 and 3. Here, the polarization maps $\alpha^{\otimes}(m \times n), \quad \beta^{\otimes}(m \times n)$ and histograms $G\left(\alpha^{\otimes}\right)$, $G\left(\beta^{\otimes}\right)$ are presented.

These data show (Figs. 2 and 3), that for all the histological sections of samples from both groups realized are various two-dimensional (fragments (1), (3)) and statistical (fragments (2), (4)) distributions of polarization parameters of autofluorescent images.

The comparative analysis of polarization maps for autofluorescent images of histological layers of myocardium revealed certain difference between them. The histograms $G\left(\alpha^{\otimes}\right)$ of the histological section of ACI are characterized by asymmetric structure (Fig. 2, fragments (2), (4)). For ACI process detected by the following changes of $Z_{i=1 ; 2 ; 3 ; 4}\left(\alpha^{\otimes}\right)$, there is the decrease of mean $Z_{1} \downarrow$ and dispersion $Z_{2} \downarrow$ of histograms
$G\left(\alpha^{\otimes}\right)$. Statistical moments (skewness $Z_{3} \uparrow$, kurtosis $\left.Z_{4} \uparrow\right)$ are increased.

For coordinate distributions of the polarization ellipticity $\beta^{\otimes}(m \times n)$, autofluorescence images found that the halfwidth of distributions $G\left(\beta^{\otimes}\right)$ for the sample of the group 2 is 1.5 times higher than the histogram for the group 1 sample (Fig. 3, fragments (2), (4)). This transformation of the statistical structure of the polarization ellipticity is accompanied by following changes of $Z_{i=1 ; 2 ; 3 ; 4}\left(\beta^{\otimes}\right)-Z_{1} \uparrow, Z_{2} \uparrow, Z_{3} \downarrow, Z_{4} \downarrow$.

\subsection{Statistical intergroup analysis}

The diagnosis of CHD (group 1) and ACI (group 2) was determined by the gold standard method. By means of software product Statmate for $95 \%$ confidence interval $(p \prec 0.05)$ a reliable quantity of people was determined $-n=52$.
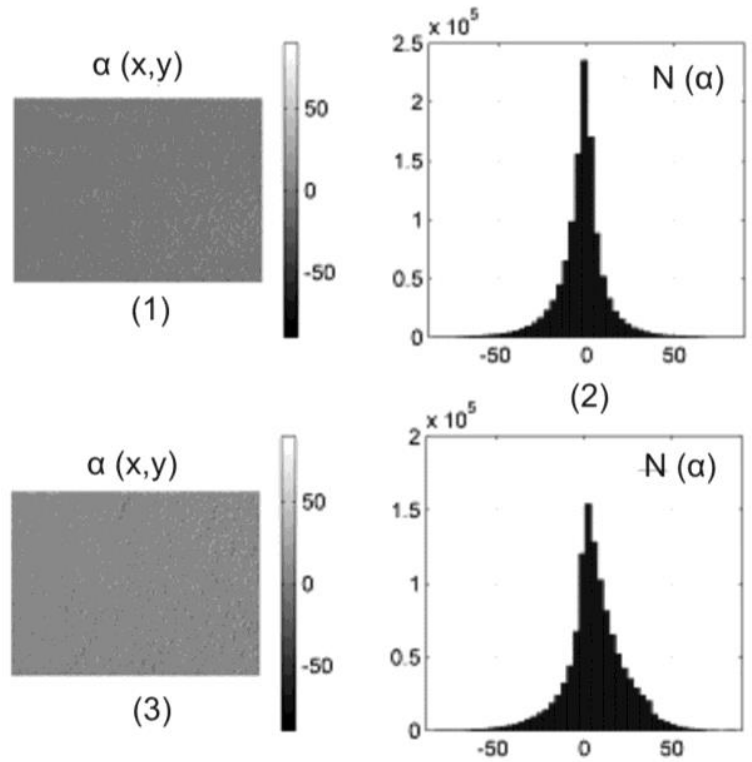

(4)

Fig. 2. Polarization maps of azimuth autofluorescence imaging of myocardium with CHD ((1), (2)) and ACI ((3), (4)). 

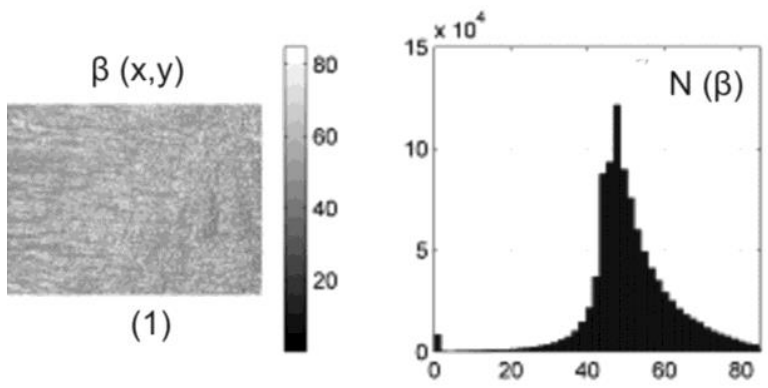

(2)
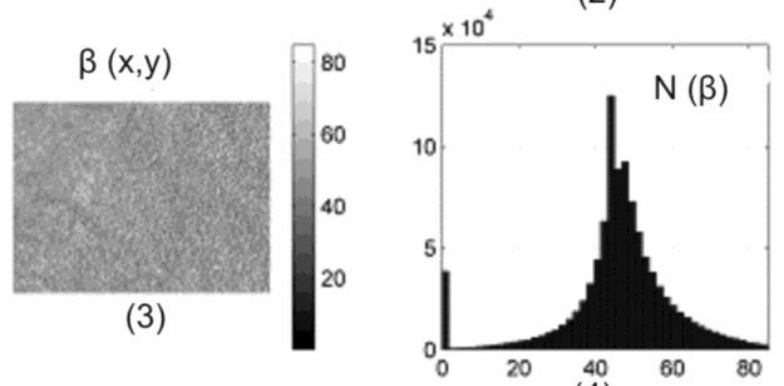

(4)

Fig. 3. Polarization maps of ellipticity autofluorescence imaging of myocardium with CHD ((1), (2)) and ACI ((3), (4)).

Determined within each group of samples are:

- $\quad$ averages of statistical moments $\bar{Z}_{i=1 ; 2 ; 3 ; 4}(q)$, their standard deviations $\pm \sigma$ and histograms $N\left(\bar{Z}_{i}\right)$ Tables 1 and 2.

- traditional characteristics for demonstrative medicine operational - sensitivity $S e=\frac{a}{a+b} 100 \%$, specificity $S p=\frac{c}{c+d} 100 \%$ and balanced accuracy $A c=\frac{S e+S p}{2}$, where $a$ and $b$ are the numbers of correct and incorrect diagnoses within the group 2; $c$ and $d$ are the same in the group 1 - Tables 3 and 4 .

Table 1. Average $\bar{Z}_{i=1 ; 2 ; 3 ; 4}\left(\alpha^{\otimes}\right)$ and standard deviations $\pm \sigma$ statistical moments $Z_{i=1 ; 2 ; 3 ; 4}$ of polarization maps of azimuth autofluorescence images distributions.

\begin{tabular}{|c|c|c|}
\hline \multirow{2}{*}{ Parameters } & \multicolumn{2}{|c|}{$\begin{array}{c}\text { Myocardium } \\
\left(n_{1}=n_{2}=52\right)\end{array}$} \\
\cline { 2 - 3 } & Group 1 & Group 2 \\
\hline$Z_{1}$ & $1.31 \pm 0.29$ & $1.13 \pm 0.23$ \\
\hline$Z_{2}$ & $0.92 \pm 0.15$ & $0.86 \pm 0.14$ \\
\hline$Z_{3}$ & $1.18 \pm 0.21$ & $1.61 \pm 0.39$ \\
\hline$Z_{4}$ & $0.94 \pm 0.14$ & $1.23 \pm 0.27$ \\
\hline
\end{tabular}

Table 2. Average $\bar{Z}_{i=1 ; 2 ; ; ; 4}\left(\beta^{\otimes}\right)$ and standard deviations $\pm \sigma$ statistical moments $Z_{i=1 ; 2 ; 3 ; 4}$ of polarization maps of ellipticity autofluorescence images distributions.

\begin{tabular}{|c|c|c|}
\hline \multirow{2}{*}{ Parameters } & \multicolumn{2}{|c|}{$\begin{array}{c}\text { Myocardium } \\
\left(n_{1}=n_{2}=52\right)\end{array}$} \\
\cline { 2 - 3 } & Group 1 & Group 2 \\
\hline$Z_{1}$ & $0.33 \pm 0.056$ & $0.37 \pm 0.061$ \\
\hline$Z_{2}$ & $0.46 \pm 0.11$ & $0.64 \pm 0.15$ \\
\hline$Z_{3}$ & $0.81 \pm 0.16$ & $0.72 \pm 0.14$ \\
\hline$Z_{4}$ & $1.61 \pm 0.39$ & $1.16 \pm 0.24$ \\
\hline
\end{tabular}

Table 3. Operational characteristics of the autofluorescence laser polarimetry azimuths method.

\begin{tabular}{|c|c|c|c|}
\hline & $Z_{i}$ & \multicolumn{2}{|c|}{ Biopsy $\left(n_{1}=n_{2}=52\right)$} \\
\hline \multirow{3}{*}{$A c\left(Z_{i}\right), \%$} & $Z_{1}$ & 59.6 & $\begin{array}{l}a=32, b=20 \\
c=30, d=22\end{array}$ \\
\cline { 2 - 4 } & $Z_{2}$ & 58.65 & $\begin{array}{l}a=31, b=21 \\
c=30, d=22\end{array}$ \\
\cline { 2 - 4 } & $Z_{3}$ & 85.55 & $\begin{array}{l}a=45, b=7 \\
c=44, d=8\end{array}$ \\
\cline { 2 - 4 } & $Z_{4}$ & 86.55 & $\begin{array}{l}a=46, b=6 \\
c=44, d=8\end{array}$ \\
\hline
\end{tabular}

Table 4. Operational characteristics of the autofluorescence laser polarimetry ellipticity method.

\begin{tabular}{|c|c|c|c|}
\hline & $Z_{i}$ & \multicolumn{2}{|c|}{ Biopsy $\left(n_{1}=n_{2}=52\right)$} \\
\hline \multirow{3}{*}{$A c\left(Z_{i}\right), \%$} & $Z_{1}$ & 55.75 & $\begin{array}{l}a=30, b=22 \\
c=28, d=24\end{array}$ \\
\cline { 2 - 4 } & $Z_{2}$ & 88.3 & $\begin{array}{l}a=47, b=5 \\
c=45, d=7\end{array}$ \\
\cline { 2 - 4 } & $Z_{3}$ & 59.6 & $\begin{array}{l}a=32, b=20 \\
c=30, d=22\end{array}$ \\
\cline { 2 - 4 } & $Z_{4}$ & 90.4 & $\begin{array}{l}a=48, b=4 \\
c=46, d=6\end{array}$ \\
\hline
\end{tabular}

The following quantitative difference between $\bar{Z}_{i=1 ; 2 ; 3 ; 4}\left(\alpha^{\otimes}, \beta^{\otimes}\right)$ was determined - the difference between statistical moments $\bar{Z}_{i=1 ; 2 ; 3 ; 4}$

$\int \alpha^{\otimes} \leftrightarrow \Delta Z_{1}=1.16, \Delta Z_{2}=1.07, \Delta Z_{3}=1.36, \Delta Z_{4}=1.31 ;$ $\left\{\beta^{\otimes} \leftrightarrow \Delta Z_{1}=1.12, \Delta Z_{2}=1.39, \Delta Z_{3}=1.13, \Delta Z_{4}=1.39\right.$.

The obtained results suggest a rather high level of accuracy obtained using this method. According to the criteria of demonstrative medicine parameters are $A c\left(Z_{3 ; 4}\left(\alpha^{\otimes}\right)\right) \sim 85 \%, A c\left(Z_{3 ; 4}\left(\beta^{\otimes}\right) \sim 90 \%\right)$. 


\section{Conclusions}

1. Being based on the model of generalized optical anisotropy inherent to myocardium tissue, the analytically grounded and experimentally tested method of laser polarization autofluorescence has been offered.

2. Within the applied approach, interrelations between the statistical moments characterizing polarizations maps of laser autofluorescent images of histological sections taken from myocardium and the peculiarities of its pathological states have been found.

3. Clinical efficiency of the technique based on polarization of laser autofluorescence observed in myocardium samples for the task of posthumous diagnosis has been demonstrated.

\section{Acknowledgement}

This work was supported by the grants № 0113U003239 and № 0112U002336 from the Ukrainian Foundation for Basic Researches.

\section{References}

1. T.T. Tower, R.T. Tranquillo, Alignment maps of tissues: I. Microscopic elliptical polarimetry // Biophys. J. 81, p. 2954-2963 (2001).

2. M. Shribak, R. Oldenbourg, Techniques for fast and sensitive measurements of two-dimensional birefringence distributions // Appl. Opt. 42 , p. 3009-3017 (2003).

3. M.H. Smith, Interpreting Mueller matrix images of tissues // Proc. SPIE, 4257, p. $82-89$ (2001).

4. X. Wang, L.V. Wang, Propagation of polarized light in birefringent turbid media: A Monte Carlo study // J. Biomed. Opt. 7, p. 279-290 (2002).

5. O.V. Angelsky, A.Ya. Bekshaev, A.Ya. Maksimyak, P.P. Maksimyak, I.I. Mokhun, S.G. Hanson, C.Yu. Zenkova, A.V. Tyurin, Circular motion of particles suspended in a Gaussian beam with circular polarization validates the spin part of the internal energy flow // Opt. Exp. 20, p. 1135111356 (2012).

6. A.Ya. Bekshaev, O.V. Angelsky, S.G. Hanson, C.Yu. Zenkova, Scattering of inhomogeneous circularly polarized optical field and mechanical manifestation of the internal energy flows // Phys. Rev. A, 86, 023847 (2012).

7. O.V. Angelsky, P.P. Maksimyak, T.O. Perun, Dimensionality in optical fields and signals // Appl. Opt. 32, p. 6066-6071 (1993).

8. O.V. Angelsky, Yu.A. Ushenko, The degree of mutual anisotropy of biological liquid crystals net during the diagnostics of human tissues birefringence // Adv. Opt. Technol. 2010, 321275 (2010).
9. O. Quijano, Arce-Diego, Mueller matrix differential decomposition // Opt. Lett. 36, p. 19421944 (2011)

10. R. Ossikovski, A. De Martino, S. Guyot, Forward and reverse product decompositions of depolarizing Mueller matrices // Opt. Lett. 32, p. 689-691 (2007).

11. Yu.A. Ushenko, A.V. Dubolazov, V.O. Balanetskaya, A.O. Karachevtsev, V.A. Ushenko, Wavelet analysis of polarization maps of human blood plasma // Optika i Spektroskopiya, 113, p. 332-343 (2012), in Russian.

12. P.O. Angelsky, A.G. Ushenko, A.V. Dubolazov, M.I. Sidor, G.B. Bodnar, G. Koval, L. Trifonyuk, The singular approach for processing polarizationinhomogeneous laser images of blood plasma layers /// J. Opt. 15, 044030 (2013).

13. Yu.A. Ushenko, Yu.Ya. Tomka, A.V. Dubolazov, Laser diagnostics of anisotropy in birefringent networks of biological tissues in different physiological conditions // Quantum Electronics, 41(2), p. 170-175 (2011).

14. Yu.A. Ushenko, Yu.Ya. Tomka, A.V. Dubolazov, O.Yu. Telenga, Diagnostics of optical anisotropy changes in biological tissues using Müller matrix // Quantum Electronics, 41(2), p. 273-277 (2011).

15. Yu.A. Ushenko, Investigation of formation and interrelations of polarization singular structure and Mueller-matrix images of biological tissues and diagnostics of their cancer changes // J. Biomed. Opt. 16, 066006 (2011).

16. Yu.O. Ushenko, O.V. Dubolazov, A.O. Karachevtsev, M.P. Gorsky, Yu.F. Marchuk, Wavelet analysis of Fourier polarized images of the human bile // Appl. Opt. 51, p. C133-C139 (2012).

17. Y.A. Ushenko, Concerted spatial-frequency and polarization-phase filtering of laser images of polycrystalline networks of blood plasma smears // J. Biomed. Opt. 17(11), 117005 (2012).

18. O. Arteaga, S. Nichols, B. Kahr, Mueller matrices in fluorescence scattering // Opt. Lett. 37, p. 2835 2837 (2012).

19. O.V. Angelsky, P.V. Polyanskii, C.V. Felde, The emerging field of correlation optics // Optics and Photonics News, 23(4), p. 25-29 (2012).

20. O.V. Angelsky, A.G. Ushenko, D.N. Burcovets, Yu.A. Ushenko, Polarization visualization and selection of biotissue image two-layer scattering medium // J. Biomed. Opt. 10(1), 014010 (2005).

21. O.V. Angelsky, A.Ya. Bekshaev, P.P. Maksimyak, A.P. Maksimyak, S.G. Hanson, C.Yu. Zenkova, Self-diffraction of continuous laser radiation in a disperse medium with absorbing particles // Opt. Exp. 21(7), p. 8922-8938 (2013).

22. O.V. Angelsky, R.N. Besaha, I.I. Mokhun, Appearance of wavefront dislocations under interference among beams with simple wavefronts // Optica Applicata, 27(4), p. 272-278 (1997). 
23. O.V. Angelsky, A.Ya. Bekshaev, P.P. Maksimyak, A.P. Maksimyak, S.G. Hanson, C.Yu. Zenkova, Orbital rotation without orbital angular momentum: mechanical action of the spin part of the internal energy flow in light beams // Opt. Exp. 20(4), p. 3563-3571 (2012).

24. O.V. Angelsky, G.V. Demianovsky, A.G. Ushenko, D.N. Burkovets, Y.A. Ushenko, Wavelet analysis of two-dimensional birefringence images of architectonics in biotissues for diagnosing pathological changes // J. Biomed. Opt. 9(4), p. 679690 (2004).

25. O.V. Angel'skiı̌, A.G. Ushenko, S.B. Ermolenko, D.N. Burkovets, Yu.A. Ushenko, O.V. Pishak, Polarization-based visualization of multifractal structures for the diagnostics of pathological changes in biological tissues // Optika $i$ Spektroskopiya, 89(5), p. 799-804 (2000), in Russian).
26. Yu.O. Ushenko, Yu.Ya. Tomka, O.V. Dubolazov, V.O. Balanets'ka, A.V. Karachevtsev, A.P. Angelsky, Wavelet-analysis for laser images of blood plasma // AECE - Adv. in Electr. and Comput. Eng., 11(2), (2011).

27. V.T. Bachinsky, Yu.O. Ushenko, Yu.Ya. Tomka, O.V. Dubolazov, V.O. Balanets'ka, A.V. Karachevtsev, Wavelet analysis for polarization maps of networks formed by liquid biological crystals in blood plasma: statistical and fractal approaches // Semiconductor Physics, Quantum Electronics \& Optoelectronics, 13(2), p. 189-201 (2010).

28. Yu.A. Ushenko, Yu.Ya. Tomka, A.V. Dubolazov, V.A. Balanetskaya, V.P. Unguryan, N.I. Zabolotna, B.P. Oleinichenko, Mueller-matrix diagnostics of optical properties inherent to polycrystalline networks of human blood plasma // Semiconductor Physics, Quantum Electronics \& Optoelectronics, 14(1), p. 98-105 (2011). 